\title{
Percutaneous contrast-enhanced ultrasound for localization and qualitative diagnosis of sentinel lymph nodes in cutaneous malignant melanoma of lower extremities: a preliminary study
}

\author{
Rui-Qian Guo ${ }^{1}$, Xi Xiang ${ }^{1}$, Li-Yun Wang ${ }^{1}$, Bi-Hui Zhu' ${ }^{1}$, Song-Ya Huang ${ }^{1}$, Xin-Yi Tang ${ }^{1}$, Jun-Jie Chen ${ }^{2}$, \\ Li Qiu ${ }^{1}$
}

${ }^{1}$ Department of Medical Ultrasound, West China Hospital of Sichuan University, Chengdu, China; ${ }^{2}$ Plastic and Burn Surgery, West China Hospital of Sichuan University, Chengdu, China

Contributions: (I) Conception and design: L Qiu, X Xiang, RQ Guo; (II) Administrative support: LY Wang, BH Zhu, SY Huang, XY Tang; (III) Provision of study materials and patients: JJ Chen; (IV) Collection and assembly of data: X Xiang, RQ Guo; (V) Data analysis and interpretation: RQ Guo, X Xiang; (VI) Manuscript writing: All authors; (VII) Final approval of manuscript: All authors.

Correspondence to: Li Qiu. Department of Medical Ultrasound, West China Hospital of Sichuan University, No. 37 Guo Xue Xiang, Chengdu 610041, China. Email: qiulihx@scu.edu.cn.

Background: To explore the feasibility of sentinel lymph node (SLN) tracing by percutaneous contrastenhanced ultrasound (pCEUS) in patients with cutaneous malignant melanoma (CMM) and the ability to enhance patterns of SLNs in diagnosing lymph nodes (LNs) metastases.

Methods: Fifty-three patients with CMM of the lower extremities treated at our hospital were included in the study. All the participants received pCEUS preoperatively. The enhanced lymphatic channels (LCs) and associated SLNs were observed and tracked in real-time. The number of enhanced LCs and enhancing patterns of SLNs were recorded. Subsequently, SLNs localized by pCEUS were pathologically examined.

Results: Of the 53 cases, SLNs were successfully localized by pCEUS in 48 cases. In total, there were 59 detected SLNs averaging $1.23 \pm 0.42$ SLNs per case. The main lymphatic drainage patterns (LDPs) were the following: one enhanced LC pointed to one or more than one SLN, and multiple enhanced LCs pointed to one or multiple SLNs. There were four enhancing patterns of SLNs (uniform, annular, uneven, and no enhancement), among which the first two were considered benign nodes, while the latter two were considered metastatic nodes. With pathological results as the gold standard, the diagnostic sensitivity and specificity by pCEUS were $90.9 \%$ and $75.0 \%$, respectively.

Conclusions: Contrast-enhanced ultrasound (US) is a feasible approach for SLN identification in patients with CMM of the lower extremities. Enhancing patterns of SLNs may help predict metastasizing SLNs. This novel method may be a promising technique for clinical application.

Keywords: Ultrasound; sentinel lymph node (SLN); cutaneous malignant; melanoma; percutaneous; contrastenhanced

Submitted Mar 05, 2021. Accepted for publication Jun 21, 2021.

doi: 10.21037/qims-21-249

View this article at: https://dx.doi.org/10.21037/qims-21-249 


\section{Introduction}

Cutaneous malignant melanoma (CMM) is a malignant tumor occurring in melanocytes in the basal layer of the epidermis, whose morbidity and mortality are increasing globally (1). It has been reported that CMM is becoming one of the cancers with the fastest-growing incidence, thus resulting in a growing public health burden worldwide (2). Besides endogenous risk factors, such as fair skin type or genetic predisposition for forming multiple nevi, exposure to ultraviolet light is the most important exogenous risk factor (3). CMM is known to spread via the lymphatic system primarily. Therefore, the presence or absence of metastatic regional lymph nodes (LNs) is of obvious importance for staging, prognosis, and determination of the treatment means for the patient $(4,5)$.

In 1977, Cabanas first reported the presence of sentinel lymph nodes (SLN) in penile carcinoma and its significance in clinical treatment (6). In the early 1990s, identification and SLN biopsy (SLNB) in clinical node stage 0 (N0) cutaneous melanoma was first reported by Morton et al. (7). The SLN is defined as the first node in the regional LN drainage basin to drain the lymph fluid around the primary tumor. Tumor cells first metastasize to the SLN and then to the next LN level. With no histological evidence of SLN metastases, the risk of regional lymphatic spread is small. At present, standard treatment strategies include wide excision of the primary tumor and SLNB to assess the status of the regional nodal basin(s) $(8,9)$.

Identification and location of the SLN is a prerequisite for SLNB. The combined application of biological dyes and radionuclides is the current gold standard for localizing the SLN $(10,11)$. The standard protocol involves preoperative lymphoscintigraphy using an injection of radiopharmaceuticals and intraoperative injection of vital blue (VB) dye. VB provides visual identification of draining lymphatic channels (LCs) and SLNs. An intraoperatively hand-held gamma probe is used to identify radioactive LNs. SLNs are identified through the colocalization of radiotracer and VB dye uptake (12). However, there are some drawbacks to this method. First, Technetium sulfur colloid and VB can identify secondary echelon LNs, resulting in unnecessary extensive nodal dissection (13). Secondly, radioactive materials increase the risk of radiation exposure and add to medical costs. Thirdly, lymphoscintigraphy cannot provide information regarding the presence of metastatic infiltration in the SLNs (12). Hence, some studies have been focusing on new sentinel node tracer technology, for example, superparamagnetic iron oxide (SPIO) nanoparticles $(14,15)$ and indocyanine green (ICG) fluorescence imaging (16).

Over recent years, percutaneous contrast-enhanced ultrasound (pCEUS) has been extensively investigated to evaluate SLNs in breast cancer, revealing that SLCs and SLNs could be preoperatively detected with high accuracy (17-19). Nevertheless, to the best of our knowledge, there are few studies on the evaluation of SLN in CMM using pCEUS, and these studies draw inconsistent conclusions. Animal studies showed that pCEUS could detect SLNs in CMM and depict metastases within the SLN (12,20-22). However, the only research that included humans did not support the use of pCEUS to detect SLNs in humans (23). Therefore, more studies are needed to clarify the role of pCEUS in the SLN evaluation of CMM.

\section{Methods}

The study was conducted following the Declaration of Helsinki (as revised in 2013). Furthermore, our study was approved by the West China Hospital of Sichuan University Ethics Committee, and written informed consent was obtained from all patients.

\section{Study sample}

Patients with CMM of the lower extremities who were scheduled for surgery and SLNB at the West China Hospital of Sichuan University between April 2019 and October 2020 were prospectively recruited into the study. Exclusion criteria included: multiple tumors, pregnancy, allergy to the contrast agent, $\mathrm{LN}$ involvement by physical examination, clinical suspicion of extranodal dissemination, and severe concomitant disease. The diagnosis of primary cutaneous melanoma was confirmed by a pathologic specimen.

\section{Ultrasound (US) examination}

All patients underwent US examination one day before surgery, which was performed by an experienced sonographer. A Philips iU22 scanner (Amsterdam, The Netherlands) equipped with high-frequency linear array probes $(9-3 \mathrm{MHz}$ ) and contrast pulse sequences (CPS) was used. Low mechanical index (MI) values were applied (MI: 0.06) to reduce microbubble destruction. The patient remained supine during the examination with adequate 
leakage of the affected lower extremities and bilateral groin. The relevant LN region was examined using a gray-scale US before injection of the contrast agent to get the general idea of the position of LNs. In addition, the popliteal fossa and the groin of the ipsilateral side of the primary tumor were explored. Meanwhile, the contralateral groin was routinely explored.

The microbubbles (SonoVue, Bracco, Milan, Italy) used as a contrast agent were reconstituted with $5 \mathrm{~mL}$ of saline $(\mathrm{NaCl} 0.9 \%)$ and fully oscillated for use. As shown in Figure 1, $0.5 \mathrm{~mL}$ of SonoVue suspension was subcutaneously injected at each of four symmetric points $5 \mathrm{~mm}$ around the tumor or surgical incision (total of $2.0 \mathrm{~mL}$ ). The injection site was continuously massaged for at least one minute to promote the drainage of the contrast agent to the LCs. Then, the area was examined for enhanced LNs and LCs draining from the injection site toward the regional $\mathrm{LN}$ basin. The first or first group of LNs along the enhanced LCs was

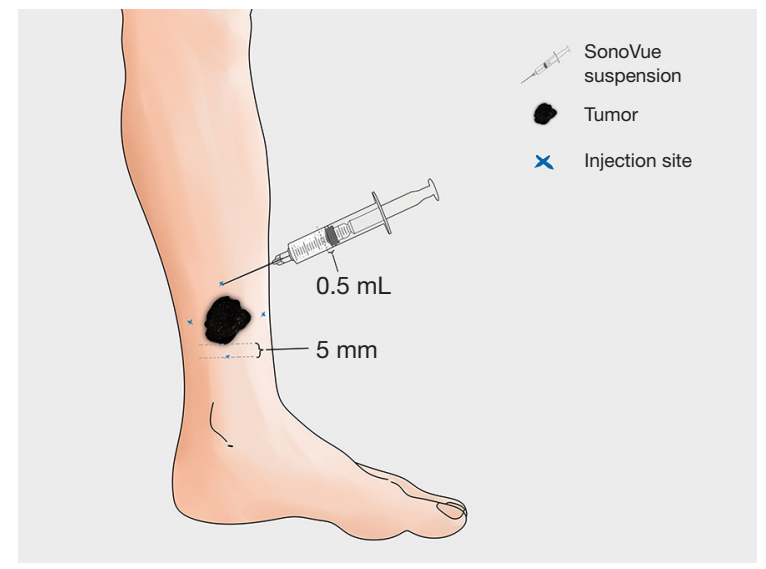

Figure 1 Scheme map for the injection method. considered as SLN/s (Figure 2).

Meanwhile, lymphatic drainage patterns (LDPs) were observed. The corresponding location of the SLNs was marked on the body surface for SLNB purposes. The scanning lasted for $5 \mathrm{~min}$, and if no enhanced LCs or LNs were found, SLN localization was considered as having failed.

\section{Treatment options}

Routine surgery was then performed with SLNB and wide local excision (WLE). The marked SLNs were sent separately for pathological examination by hematoxylineosin (HE) and immunohistochemistry (IHC). The following indicators were used: S-100, HMB45, MelanA, MART-1, MITF, and Ki-67. Finally, a completion LN dissection (CLND) was performed if the SLNs were positive for metastases.

The postoperative follow-up evaluation included physical examination, chest X-ray, and US assessment of the regional nodal basins. In addition, further investigations, including computed tomography (CT) scan, magnetic resonance imaging (MRI), and/or positron emission tomography (PET) scan were also performed selectively to confirm abnormal findings suggestive of metastatic melanoma.

\section{Statistical analysis}

The Statistical Program for the Social Sciences (SPSS) software (Version 20.0, IBM, Armonk, NY, USA) was used for statistical analysis. The corresponding statistical description and analysis methods were selected according to the data distribution. The pathological results were used as the gold standard to calculate the sensitivity and specificity

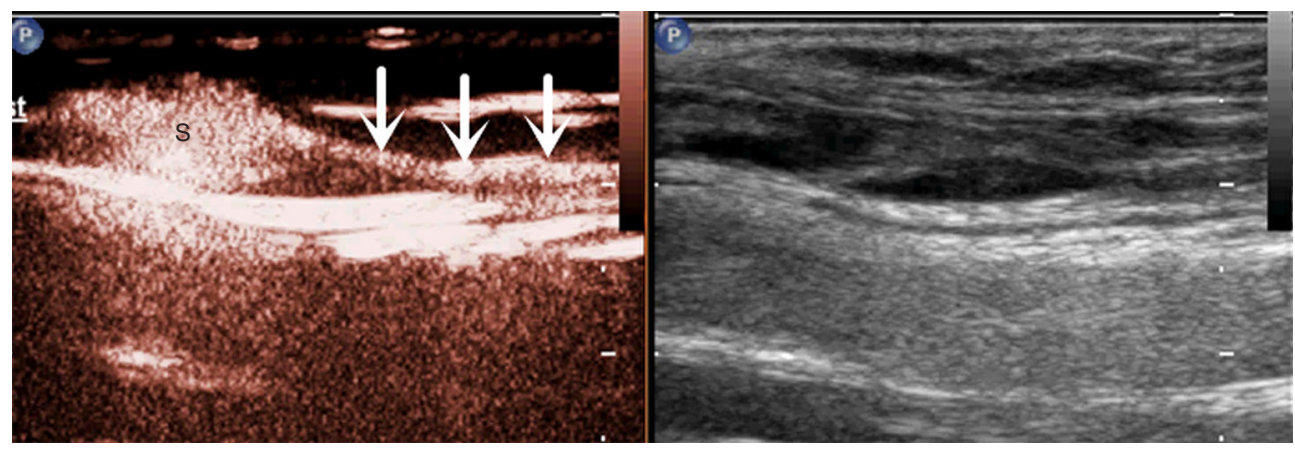

Figure 2 pCEUS identified SLN (S) along with enhanced LC (arrows). pCEUS, percutaneous contrast-enhanced ultrasound; SLN, sentinel lymph node; LC, lymphatic channel. 
Table 1 Demographic and clinical characteristics of $53 \mathrm{CMM}$ patients

\begin{tabular}{lc}
\hline Characteristic & $\mathrm{n}$ \\
\hline Age, y, mean $\pm \mathrm{SD}$ & $59 \pm 15$ \\
Gender, female: male & $29: 24$ \\
Height, cm, mean $\pm \mathrm{SD}$ & $159.6 \pm 8.7$ \\
Weight, kg, mean $\pm \mathrm{SD}$ & $62.8 \pm 11.5$ \\
Patients, $\mathrm{n}(\%)$ & \\
First WLE & $19(35.85)$ \\
Second WLE & \\
Location distribution, $\mathrm{n}(\%)$ & $34(64.15)$ \\
Thigh & $2(3.77)$ \\
Lower leg & $3(5.66)$ \\
Knee & $1(1.89)$ \\
Ankle & $1(1.89)$ \\
Foot & $46(86.79)$ \\
\hline
\end{tabular}

${ }^{a}$, insufficient margin distance for the first operation. CMM, cutaneous malignant melanoma; y, years; cm, centimeter; kg, kilogram; SD, standard deviation; WLE, wide local excision.

of pCEUS for the diagnosis of SLN metastasis. Fisher's exact test was used to compare the difference in recurrence or metastasis rates between the two groups (patients with negative SLNs vs. patients with positive SLNs). A two-sided $P$ value less than 0.05 was considered as indicating statistical significance.

\section{Results}

\section{Patients}

From April 2019 to October 2020, 53 selected patients with primary cutaneous melanoma were enrolled to undergo pCEUS studies before performing WLE and SLNB at the West China Hospital of Sichuan University. Clinical characteristics of the study sample are presented in Table 1. The mean age was $59 \pm 15$ years, and 29 patients were female. The average height was $159.6 \pm 8.7 \mathrm{~cm}$, and the average weight was $62.8 \pm 11.5 \mathrm{~kg}$. In our study population, 46 patients had a cutaneous melanoma on the foot, 3 patients had melanoma on the lower leg, 2 patients on one of the thighs, 1 patient on one of the knees, and 1 patient on one of the ankles.

\section{$p C E U S$}

No adverse reactions occurred during the pCEUS examination and within one week after the examination. In 48 out of $53 \mathrm{CMM}$ cases, enhanced LC draining from the primary lesion was clearly visualized, and the SLNs that enhanced LCs were located. The identification rates of pCEUS for SLNs were 90.6\% (48/53). In the 48 successful cases, only one SLN was detected on pCEUS in 37 cases (77.1\%), and 11 cases (22.9\%) had two visualized SLNs. In total, 59 SLNs were detected by pCEUS averaging $1.23 \pm 0.42$ SLNs per case. All SLNs were located in the ipsilateral inguinal region. Regarding the remaining 5 cases, there were no enhanced LCs or LNs in 4 of them, while a short segment of a lymphatic vessel was identified in 1 patient.

\section{LDP detection by pCEUS}

In this study, we also observed the pattern of lymphatic drainage. The following main patterns were identified (Figures 3,4): (I) one enhanced LC pointed to one SLN, which was the most common pattern (21 cases); (II) one enhanced LC pointed to more than one SLN (4 cases); (III) multiple enhanced LCs pointed to one SLN (15 cases); (IV) multiple enhanced LCs pointed to multiple SLNs (8 cases).

\section{Enhancing pattern of pCEUS}

According to different enhancement features of SLNs, we divided enhancing patterns into the following four types: (I) type 1, overall uniform enhancement (Figure 5A); (II) type 2, annular enhancement with low or no center enhancement (Figure 5B); (III) type 3, overall uneven enhancement with a mixture of high and low enhancement (Figure 5C); and (IV) type 4 , no enhancement or weak enhancement of the node connected with enhanced LCs (Figure 5D). Among the 59 SLNs detected in our study, there were 11 of type $1(18.6 \%)$, 26 of type $2(44.1 \%), 16$ of type $3(27.1 \%)$, and 6 of type 4 $(10.2 \%)$.

\section{SLNs qualitatively assessed by pCEUS}

Among four pCEUS enhancing patterns, type 1 and type 2 were considered benign SLNs, and type 3 and type 4 were considered metastatic SLNs. Hence, CEUS findings indicated 37 negative SLNs and 22 positive SLNs. 
A

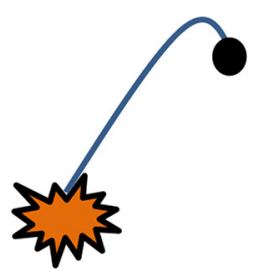

C

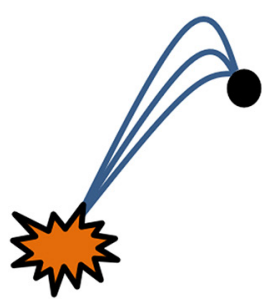

B

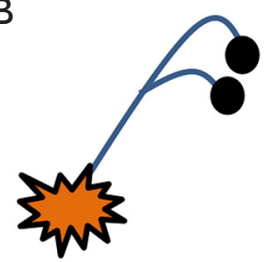

D

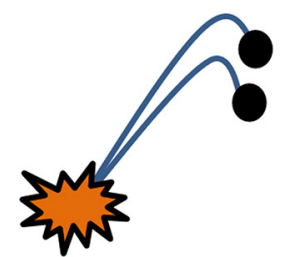

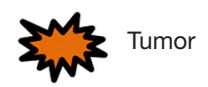

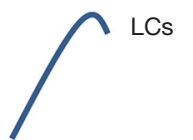

SLNs

Figure 3 Lymphatic drainage patterns in 4 types. (A) One enhanced LC pointed to one SLN. (B) One enhanced LC pointed to more than one SLN. (C) Multiple enhanced LCs pointed to one SLN. (D) Multiple enhanced LCs pointed to multiple SLNs. LC, lymphatic channel; SLN, sentinel lymph node.

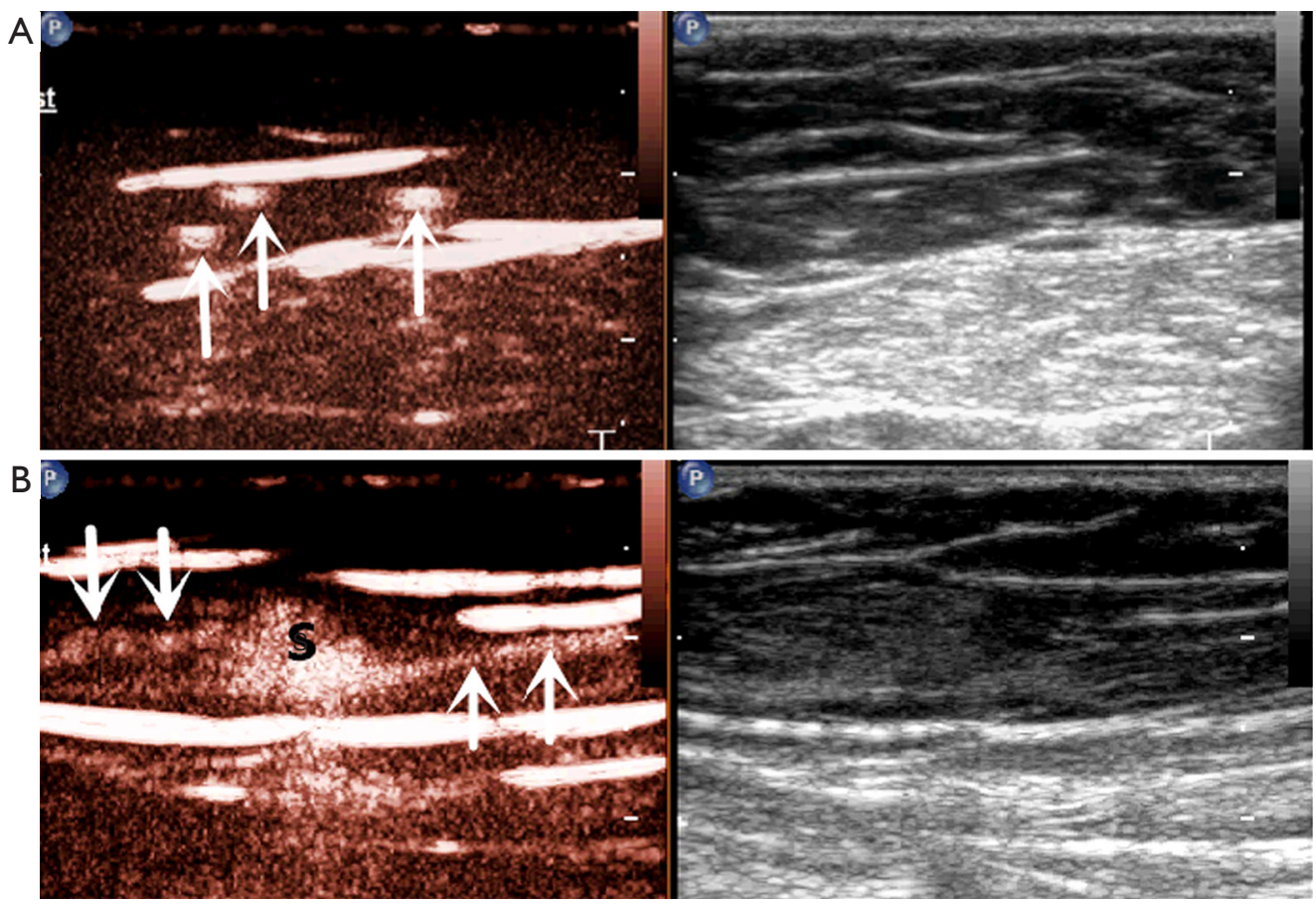

Figure 4 Multiple enhanced LCs (arrows) pointed to one SLN (S). (A) Cross section of LCs. (B) Longitudinal section of LCs. LC, lymphatic channel; SLN, sentinel lymph node. 

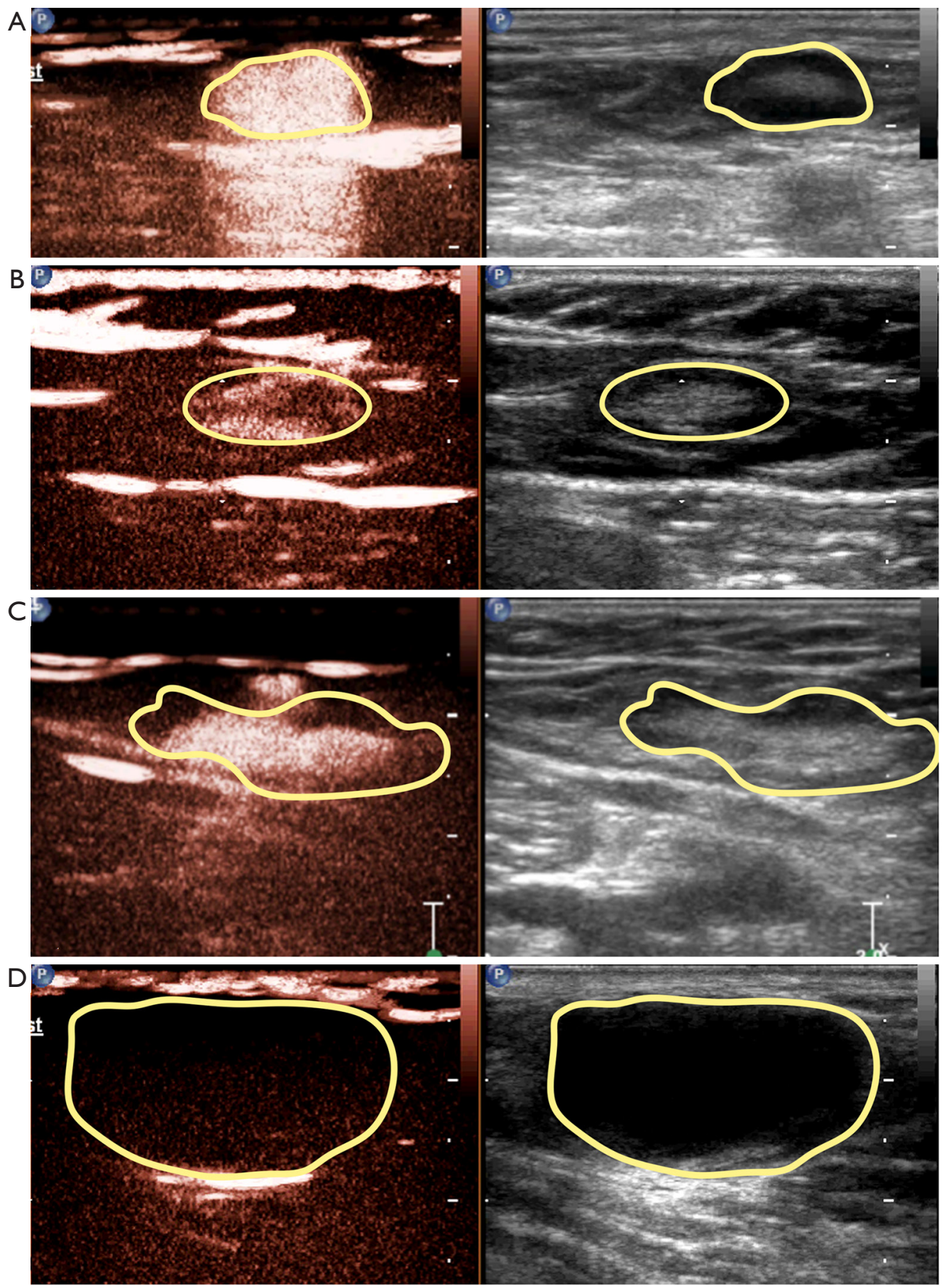

Figure 5 pCEUS enhancement of SLNs in 4 types. (A) Overall uniform enhancement. (B) Annular enhancement with low or no center enhancement. (C) Overall uneven enhancement with a mixture of high and low enhancement. (D) No enhancement or weak enhancement of the node connected with enhanced LCs. The yellow circles indicate the marginal outline of the lymph node. pCEUS, percutaneous contrast-enhanced ultrasound; SLN, sentinel lymph node; LC, lymphatic channel. 
Table 2 Pathological and percutaneous contrast-enhanced ultrasound pCEUS diagnosis of 59 SLNs

\begin{tabular}{lccc}
\hline \multirow{2}{*}{ pCEUS } & \multicolumn{2}{c}{ Pathology } & Total \\
\cline { 2 - 3 } & Positive & Negative & \\
\hline Positive & 10 & 12 & 22 \\
Negative & 1 & 36 & 37 \\
Total & 11 & 48 & 59 \\
\hline
\end{tabular}

pCEUS, percutaneous contrast-enhanced ultrasound; SLN, sentinel lymph node.

According to pathological diagnosis, there were 48 negative SLNs and 11 positive SLNs. Thus, the sensitivity and specificity of CEUS for the diagnosis of SLN were $90.9 \%$ $(10 / 11)$ and $75.0 \%(36 / 48)$, respectively (Table 2).

\section{Post-surgical follow-up}

Thirty-two of the 53 patients were followed up successfully, including 21 patients with positive SLNs and 11 patients with negative SLNs defined by pCEUS. The median follow-up time was 7 months (range, 1-16 months). In the positive group, 4 patients developed distant metastases (lung, bone), and 1 patient had regional $\mathrm{LN}$ recurrence. The rate of recurrence or metastases in the SLNs positive group was $45.5 \%(5 / 11)$. In the negative group, 1 patient developed surgical site recurrence, and 1 patient developed pulmonary metastasis, but no regional LN abnormality was found. The rate of recurrence or metastases in SLNs in the negative group was $9.5 \%(2 / 21)$. The difference between the two groups was statistically significant $(\mathrm{P}<0.05)$.

\section{Discussion}

CMM is the most aggressive type of skin cancer, accounting for $90 \%$ of all skin cancer-related mortalities (24). Although melanoma incidence remains low in China, it has rapidly increased, with approximately 20,000 new cases reported each year (25). Among Chinese people, primary melanoma of the extremities accounts for about $50 \%$ of primary melanomas, where the most common primary sites include feet, fingers, and lower extremities (26). Therefore, as a preliminary study, we only included patients with CMM of the lower extremities.

LN status is the most important prognostic factor in CMM. Current guidelines for treating cutaneous melanoma recommend SLNB for melanomas with Breslow depth greater than $1 \mathrm{~mm}$ or tumors with less than $1 \mathrm{~mm}$ in depth and with certain high-risk features, followed by CLND, if the SLN is tumor positive (10). The standard protocol for SLNB involves preoperative lymphoscintigraphy using an injection of radiocolloid, followed by intraoperative LN mapping using blue dye. According to reports in the literature, blue dye and radiocolloid particles can pass through the first echelon LNs due to their small size (20), leading to more extensive dissection and increased complications (27). Blue dye is often avoided in facial melanoma for esthetic and surgical reasons (28). On the other hand, due to the need for preoperative preparation or intraoperative operation, these methods may increase the operation time and morbidity. Given the situation described above, new techniques for finding SLNs are needed.

SLN biopsy done by pCEUS, which has been a focus of research over recent years, especially for breast cancer, has shown a promising performance (29). However, only a few studies focused on percutaneous pCEUS in SLN in CMM. Also, the only human study did not support its use in the SLNs in CMM (23). Microbubble-based US contrast agents, such as Sonazoid and Sonovue, have a mean bubble diameter of $2.5 \mu \mathrm{m}$, which is much less than red blood cells (mean $7.2 \mu \mathrm{m}$ ). Consequently, the bubbles can easily pass through the blood capillary and LCs (17). Also, studies have shown that the contrast enhancement of the lymphatic system was confined within the first draining LN (i.e., the SLNs) without passing to the next tier of the LN (20-22,30). The safety of pCEUS has been confirmed in animal and human studies $(17,23)$.

In our study, the detection rate of SLN was 48/53, which demonstrates the feasibility of SLN detection with pCEUS in patients with CMM, and which is similar to results reported for patients with breast cancer $(17,19)$. All SLNs detected by pCEUS were located at the groin, consistent with previous reports on SLNs of CMM in the distal lower extremities found in the ipsilateral inguinal region (31). Among the other 5 patients with no detected SLNs, 4 had abnormal signs on gray-scale ultrasound; thus, regional CLND was completed, and postoperative pathology confirmed LN metastases. Consequently, we speculated that detection failed because LCs were blocked by tumor cells. It is worth noting that among these 4 patients, 2 developed popliteal LN metastases, suggesting that the SLNs of CMM could be located in the popliteal fossa (31). As for another patient with no detected SLN, one enhanced LC was visualized, but follow-up failed before drainage to any $\mathrm{LN}$. Also, no metastatic LNs were found on regional CLND, 
which may be due to the use of an inadequate contrast dose.

Our results are contrary to Rue Nielsen et al., whose study did not support the use of pCEUS for the detection of SNs in CMM in humans due to the low detection rate (23). Based on our analysis, we assume this might be related to the following factors: (I) the number of spots in a subcutaneous injection (in our study, we selected four points around the lesion rather than two points); (II) whether adequate local massage was performed before scanning [it was reported that massaging the peritumoral area resulted in a qualitatively appreciable increase in the rate of flow of contrast agent within the LCs and a more rapid enhancement of the SLNs (20)]; and (III) the lower mechanical index values applied in our study (MI: 0.06 vs. 0.13). Also, the destruction of microbubbles may be reduced to a greater extent.

We also found multiple LDPs in this study, which has also been reported in previous animal models $(21,22)$. This prompted us to enlarge the scanning area when tracking the enhanced LCs, to avoid missing SLNs. Furthermore, in an animal model, the number of enhanced LCs was reported to be associated with the number of injection points, and multiple injection points increased the number of enhanced LCs, where lymphatic drainage from each site led to the SLN(s) (20). Hence, we thought that SLNs were more accurately detected by multipoint injection than by singlesite injection.

Another advantage of SLNs localization by US was that the enhancing pattern could preliminarily predict the state of the SLNs. In this paper, we observed four enhancing patterns of SLNs. The first type was the uniform enhancement of the entire $\mathrm{LN}$, which suggests no metastasis in the LN. Also, the contrast agent was distributed evenly within the LN $(17,18,20,22)$. The second type was an annular enhancement, which we classified as a benign sign. As is well known, the normal direction of internal drainage of LNs is from the cortex to cortex, which appears to be enhanced in pCEUS from the peripheral region to the central zone (17).

Consequently, we speculated that this might be due to the long distance from the lesion to the regional $\mathrm{LN}$, resulting in a relatively insufficient contrast dose. Considering that no adverse reactions occurred in this study, the contrast agent dose should be increased as appropriate in future studies. The third type was an overall uneven enhancement. The previous study supported that SLNs that contained metastases demonstrated areas of enhancement (normal LN parenchyma) and areas that were not enhanced due to tumor infiltration and displacement or destruction of normal tissue $(17,18,20)$. The last type was no enhancement, which suggests metastatic SLN. Metastases replaced the normal LN parenchyma, or the main LCs were blocked, resulting in failure of the contrast agent filling $(18,22)$.

Our study showed that the sensitivity and specificity of pCEUS for the diagnosis of SLN metastasis were $90.9 \%$ and $75.0 \%$, respectively. According to the existing literature, the lower specificity may be due to the following reasons. First, benign lesions such as lymphoid follicle hyperplasia and lymphatic sinus dilation could block lymphatic drainage, resulting in the retention of contrast media in the lymphoid parenchyma, which is characterized by uneven enhancement (32). Some previous studies have reported that the tumor thrombus blocked the end of afferent LCs, thus preventing the contrast agent from entering the SLN. However, no tumor was found in the SLN, thus showing no enhancement in pCEUS (18). Combining pCEUS with gray-scale ultrasound, Doppler ultrasound, or intravenous CEUS may be a suitable method to improve diagnostic specificity $(33,34)$, which is our next planned research topic.

There are several limitations to our study. First, we did not compare pCEUS with lymphoscintigraphy and blue dye. Still, in animal models of melanoma and human breast cancer studies, pCEUS for SLN has been reported to have good correlations with these standard detection techniques (18-22). Second, as this was a preliminary study that verified the application of pCEUS in SLNs of CMM, we only selected patients with CMM of the lower extremities with relatively fixed lymphatic reflux. The evaluation of SLNs at other sites needs further verification.

In conclusion, pCEUS could effectively identify LCs and localize SLNs for guiding SLNBs in patients with CMM of the lower extremities. Also, the enhancement pattern of SLNs can predict metastasis of SLNs with high sensitivity and rather low specificity. In further studies, patients with lesions located at other sites need to be included to clarify the practicability of the technique. pCEUS with gray-scale ultrasound, Doppler ultrasound, and intravenous CEUS should be used in combination, and their ability to improve the specificity of diagnosis of metastatic SLNs should be investigated.

\section{Acknowledgments}

Funding: This study was sponsored by the National Natural Science Foundation of China (No. 81971622) and the Sichuan Science and Technology Program (No. 
2019YFS0219).

\section{Footnote}

Conflicts of Interest: All authors have completed the ICMJE uniform disclosure form (available at https://dx.doi. org/10.21037/qims-21-249). The authors have no conflicts of interest to declare.

Ethical Statement: The authors are accountable for all aspects of the work in ensuring that questions related to the accuracy or integrity of any part of the work are appropriately investigated and resolved. The study was conducted following the Declaration of Helsinki (as revised in 2013). The study was approved by the West China Hospital of Sichuan University Ethics Committee, and written informed consent was obtained from all patients.

Open Access Statement: This is an Open Access article distributed in accordance with the Creative Commons Attribution-NonCommercial-NoDerivs 4.0 International License (CC BY-NC-ND 4.0), which permits the noncommercial replication and distribution of the article with the strict proviso that no changes or edits are made and the original work is properly cited (including links to both the formal publication through the relevant DOI and the license). See: https://creativecommons.org/licenses/by-nc-nd/4.0/.

\section{References}

1. Jemal A, Siegel R, Ward E, Murray T, Xu J, Smigal C, Thun MJ. Cancer statistics, 2006. CA Cancer J Clin 2006;56:106-30.

2. Lens MB, Dawes M. Global perspectives of contemporary epidemiological trends of cutaneous malignant melanoma. Br J Dermatol 2004;150:179-85.

3. Lodde G, Zimmer L, Livingstone E, Schadendorf D, Ugurel S. Malignant melanoma. Pathologe 2020;41:281-92.

4. Morton DL, Thompson JF, Cochran AJ, Mozzillo N, Nieweg OE, Roses DF, Hoekstra HJ, Karakousis CP, Puleo CA, Coventry BJ, Kashani-Sabet M, Smithers BM, Paul E, Kraybill WG, McKinnon JG, Wang HJ, Elashoff R, Faries MB; MSLT Group. Final trial report of sentinelnode biopsy versus nodal observation in melanoma. $\mathrm{N}$ Engl J Med 2014;370:599-609.

5. Balch CM, Gershenwald JE, Soong SJ, Thompson JF, Atkins MB, Byrd DR, Buzaid AC, Cochran AJ, Coit DG, Ding S, Eggermont AM, Flaherty KT, Gimotty PA,
Kirkwood JM, McMasters KM, Mihm MC Jr, Morton DL, Ross MI, Sober AJ, Sondak VK. Final version of 2009 AJCC melanoma staging and classification. J Clin Oncol 2009;27:6199-206.

6. Cabanas RM. An approach for the treatment of penile carcinoma. Cancer 1977;39:456-66.

7. Morton DL, Wen DR, Wong JH, Economou JS, Cagle LA, Storm FK, Foshag LJ, Cochran AJ. Technical details of intraoperative lymphatic mapping for early stage melanoma. Arch Surg 1992;127:392-9.

8. Mocellin S, Pasquali S, Nitti D. The impact of surgery on survival of patients with cutaneous melanoma: revisiting the role of primary tumor excision margins. Ann Surg 2011;253:238-43.

9. Ross MI, Gershenwald JE. Evidence-based treatment of early-stage melanoma. J Surg Oncol 2011;104:341-53.

10. Wong SL, Balch CM, Hurley P, Agarwala SS, Akhurst TJ, Cochran A, Cormier JN, Gorman M, Kim TY, McMasters KM, Noyes RD, Schuchter LM, Valsecchi ME, Weaver DL, Lyman GH; Society of Surgical Oncology. Sentinel lymph node biopsy for melanoma: American Society of Clinical Oncology and Society of Surgical Oncology joint clinical practice guideline. J Clin Oncol 2012;30:2912-8.

11. Garbe C, Peris K, Hauschild A, Saiag P, Middleton M, Spatz A, Grob JJ, Malvehy J, Newton-Bishop J, Stratigos A, Pehamberger H, Eggermont AM; European Organization of Research and Treatment of Cancer. Diagnosis and treatment of melanoma. European consensus-based interdisciplinary guideline--Update 2012. Eur J Cancer 2012;48:2375-90.

12. Bertelsen C, King KG, Swanson M, Duddalwar V, Pepper JP. Contrast-Enhanced Ultrasound With Perflubutane for Sentinel Lymph Node Mapping in Cutaneous Melanoma: A Pilot Study. Laryngoscope 2019;129:1117-22.

13. Goldfarb LR, Alazraki NP, Eshima D, Eshima LA, Herda SC, Halkar RK. Lymphoscintigraphic identification of sentinel lymph nodes: clinical evaluation of 0.22 -micron filtration of Tc-99m sulfur colloid. Radiology 1998;208:505-9.

14. Mok CW, Tan SM, Zheng Q, Shi L. Network metaanalysis of novel and conventional sentinel lymph node biopsy techniques in breast cancer. BJS Open 2019;3:445-52.

15. Wang YX, Wang DW, Zhu XM, Zhao F, Leung KC. Carbon coated superparamagnetic iron oxide nanoparticles for sentinel lymph nodes mapping. Quant Imaging Med Surg 2012;2:53-6.

16. Knackstedt R, Couto RA, Ko J, Cakmakoglu C, Wu D, 
Gastman B. Indocyanine Green Fluorescence Imaging with Lymphoscintigraphy for Sentinel Node Biopsy in Melanoma: Increasing the Sentinel Lymph Node-Positive Rate. Ann Surg Oncol 2019;26:3550-60.

17. Xie F, Zhang D, Cheng L, Yu L, Yang L, Tong F, Liu H, Wang S, Wang S. Intradermal microbubbles and contrastenhanced ultrasound (CEUS) is a feasible approach for sentinel lymph node identification in early-stage breast cancer. World J Surg Oncol 2015;13:319.

18. Liu J, Liu X, He J, Gou B, Luo Y, Deng S, Wen H, Zhou L. Percutaneous contrast-enhanced ultrasound for localization and diagnosis of sentinel lymph node in early breast cancer. Sci Rep 2019;9:13545.

19. Cox K, Sever A, Jones S, Weeks J, Mills P, Devalia H, Fish $\mathrm{D}$, Jones P. Validation of a technique using microbubbles and contrast enhanced ultrasound (CEUS) to biopsy sentinel lymph nodes (SLN) in pre-operative breast cancer patients with a normal grey-scale axillary ultrasound. Eur J Surg Oncol 2013;39:760-5.

20. Goldberg BB, Merton DA, Liu JB, Thakur M, Murphy GF, Needleman L, Tornes A, Forsberg F. Sentinel lymph nodes in a swine model with melanoma: contrast-enhanced lymphatic US. Radiology 2004;230:727-34.

21. Goldberg BB, Merton DA, Liu JB, Murphy G, Forsberg F. Contrast-enhanced sonographic imaging of lymphatic channels and sentinel lymph nodes. J Ultrasound Med 2005;24:953-65.

22. Goldberg BB, Merton DA, Liu JB, Forsberg F, Zhang K, Thakur M, Schulz S, Schanche R, Murphy GF, Waldman SA. Contrast-enhanced ultrasound imaging of sentinel lymph nodes after peritumoral administration of Sonazoid in a melanoma tumor animal model. J Ultrasound Med 2011;30:441-53.

23. Rue Nielsen K, Klyver H, Hougaard Chakera A, Nedergaard L, Hesse B, Bachmann Nielsen M. Sentinel node detection in melanomas using contrast-enhanced ultrasound. Acta Radiol 2009;50:412-7.

24. Aubuchon MM, Bolt LJ, Janssen-Heijnen ML, Verleisdonk-Bolhaar ST, van Marion A, van Berlo CL. Epidemiology, management and survival outcomes of primary cutaneous melanoma: a ten-year overview. Acta Chir Belg 2017;117:29-35.

25. Guo J, Qin S, Liang J, Lin T, Si L, Chen X, et al. Chinese Guidelines on the Diagnosis and Treatment of Melanoma (2015 Edition). Ann Transl Med 2015;3:322.
26. Chinese guidelines for diagnosis and treatment of melanoma 2018 (English version). Chin J Cancer Res 2019;31:578-85.

27. Solari N, Bertoglio S, Boscaneanu A, Minuto M, Reina S, Palombo D, Bruzzi P, Cafiero F. Sentinel lymph node biopsy in patients with malignant melanoma: analysis of post-operative complications. ANZ J Surg 2019;89:1041-4.

28. Kelly J, Fogarty K, Redmond HP. A definitive role for sentinel lymph node mapping with biopsy for cutaneous melanoma of the head and neck. Surgeon 2009;7:336-9.

29. Cui Q, Dai L, Li J, Xue J. Accuracy of CEUS-guided sentinel lymph node biopsy in early-stage breast cancer: a study review and meta-analysis. World J Surg Oncol 2020;18:112.

30. Liu JB, Merton DA, Berger AC, Forsberg F, Witkiewicz A, Zhao H, Eisenbrey JR, Fox TB, Goldberg BB. Contrastenhanced sonography for detection of secondary lymph nodes in a melanoma tumor animal model. J Ultrasound Med 2014;33:939-47.

31. Miranda SG, Parrett BM, Li RR, Lee G, Chang T, Fadaki N, Cardona-Huerta S, Cleaver JE, Kashani-Sabet M, Leong SP. Selective Sentinel Lymph Node Dissection in Lower Extremity Melanoma. Plast Reconstr Surg 2016;137:1031-8.

32. Aoki T, Moriyasu F, Yamamoto K, Shimizu M, Yamada M, Imai Y. Image of tumor metastasis and inflammatory lymph node enlargement by contrast-enhanced ultrasonography. World J Radiol 2011;3:298-305.

33. Dudea SM, Lenghel M, Botar-Jid C, Vasilescu D, Duma M. Ultrasonography of superficial lymph nodes: benign vs. malignant. Med Ultrason 2012;14:294-306.

34. Tombesi P, Tassinari D, Sartori S. Contrast-enhanced ultrasound for characterizing lymph nodes with focal cortical thickening in patients with cutaneous melanoma. AJR Am J Roentgenol 2011;197:W371; author reply W372.

Cite this article as: Guo RQ, Xiang X, Wang LY, Zhu BH, Huang SY, Tang XY, Chen JJ, Qiu L. Percutaneous contrastenhanced ultrasound for localization and qualitative diagnosis of sentinel lymph nodes in cutaneous malignant melanoma of lower extremities: a preliminary study. Quant Imaging Med Surg 2022;12(1):366-375. doi: 10.21037/qims-21-249 